\title{
Impedance Aggregometry platelet aggregation is dependent on platelet count .
}

\author{
Soliman M., Peters J., Hartmann M. \\ Klinik für Anästhesiologie und Intensivmedizin, Universität Duisburg-Essen \\ \& Universitätsklinikum Essen, Essen, Germany
}

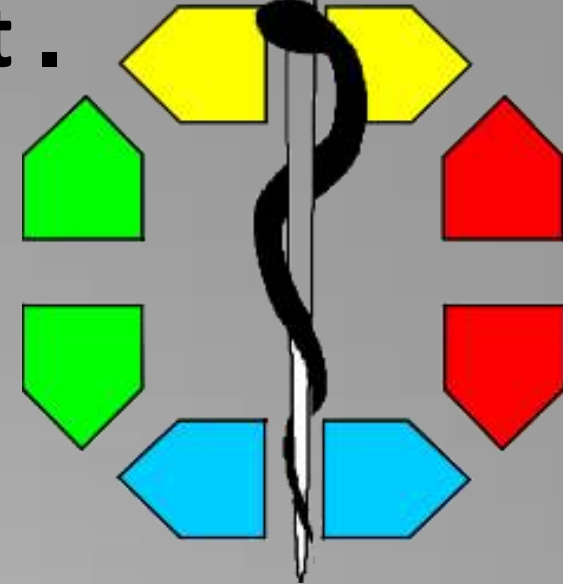

Introduction: Impedance aggregometry (Multiplate $\AA$ ) is useful to predict thrombotic complications after coronary and cerebrovascular stent interventions (1-3). However, despite poor evidence, it is often taken for granted, that platelet concentration does not affect platelet aggregation when platelet concentration is within the physiological range. We hypothesized that 1) platelet aggregation, as measured by impedance aggregometry and platelet concentration highly correlate, and 2) the aggregation/platelet count ratio reflects platelet function independent of platelet concentration.

Method: Following ethics committee approval, platelet rich plasma, derived by centrifugation with $110 \mathrm{~g}$ for 5 minutes at $24^{\circ} \mathrm{C}$ of blood samples from 8 different patients, was diluted with platelet poor plasma yielding different blood concentrations of platelets. Thereafter, platelet concentration was measured and samples were subjected to impedance aggregometry using thrombin receptor-activating peptide (TRAP) for platelet activation. Platelet poor plasma was obtained by centrifugation at $1800 \mathrm{~g}$ for 10 minutes at $24^{\circ} \mathrm{C}$. Aggregation amplitude and platelet concentration were correlated and aggregation was compared in undiluted and 2-fold diluted samples. Statistics: Pearson correlation and Student's t-test for paired sample.

Results: In every single donor experiment, platelet concentration and impedance aggregometry amplitude highly correlated (mean correlation coefficient: $0.91 \pm 0.07)$, and, in all measurements $(n=32)$, the correlation between evoked platelet aggregation amplitude and platelet concentration was $(r=0.78, p=0.0001)$, with an aggregation unit/platelet concentration correlation of $0.17(p=0.3)$. Moreover, there was a significant difference when comparing platelet aggregation in undiluted and 2 -folds diluted samples $(n=19,94.3 \mathrm{AU}$ vs $41.8, p=0.0001)$. In contrast, there was no difference in the aggregation/count ratio between undiluted and 2 -folds diluted samples $\left(n=19,0.33 \mathrm{AU} / 10^{3}\right.$ platelet $\mathrm{mm}^{-3}$ vs $\left.0.27, p=0.13\right)$.
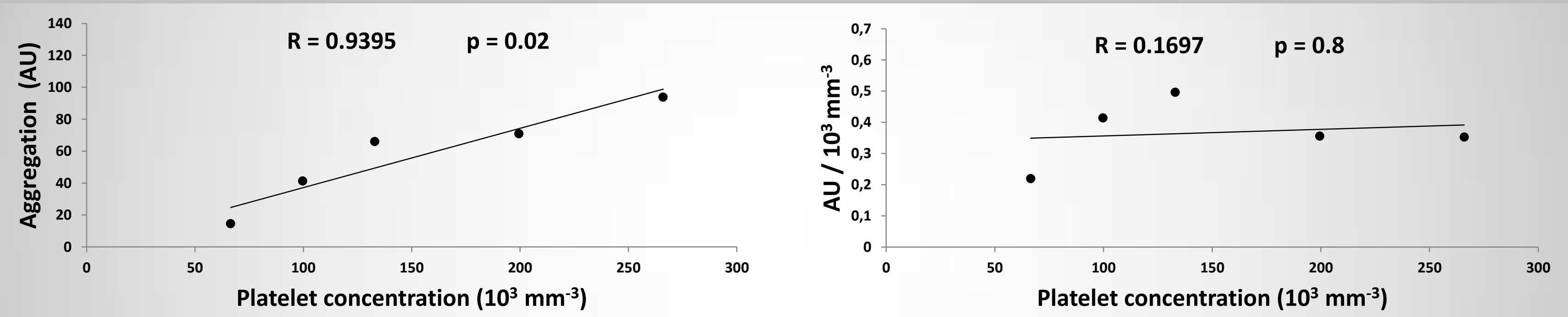

-Correlation between Aggregometry amplitude and platelet concentration (left side) and correlation of the aggregation/platelet concentration ratio and platelet concentration (right side) in blood from one of the individuals.

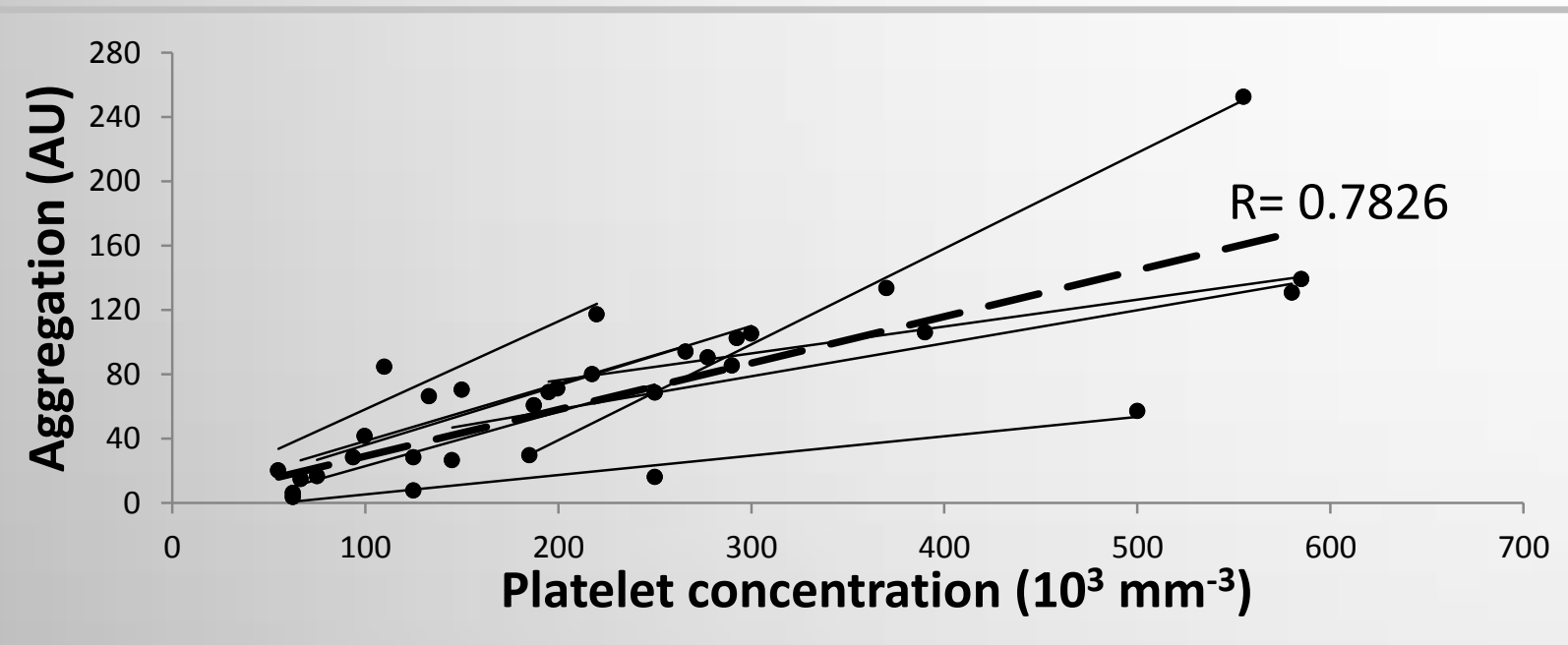

Correlation between Aggregometry amplitude and platelet concentration with 32 data points from 8 indidividuals.
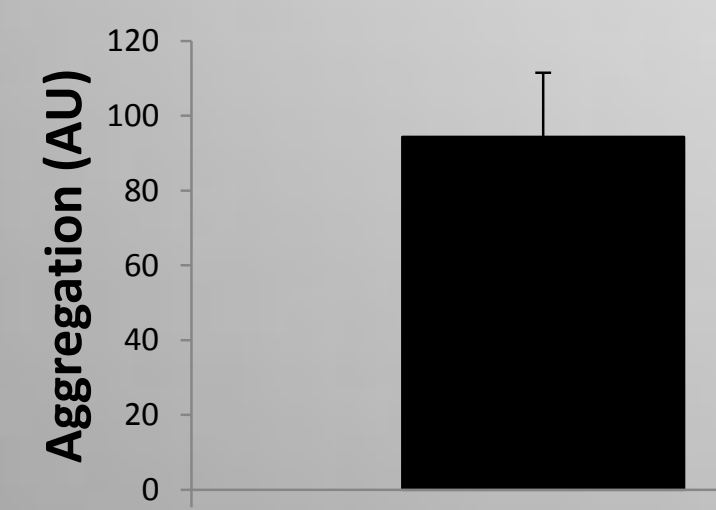

undiluted

$p=0.0001$

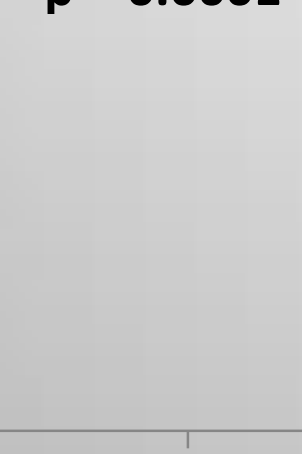

(RAP

Comparison between TRAP evoked aggregation in undiluted $n=19$ and twofold diluted platelet rich plasma $n=19$.

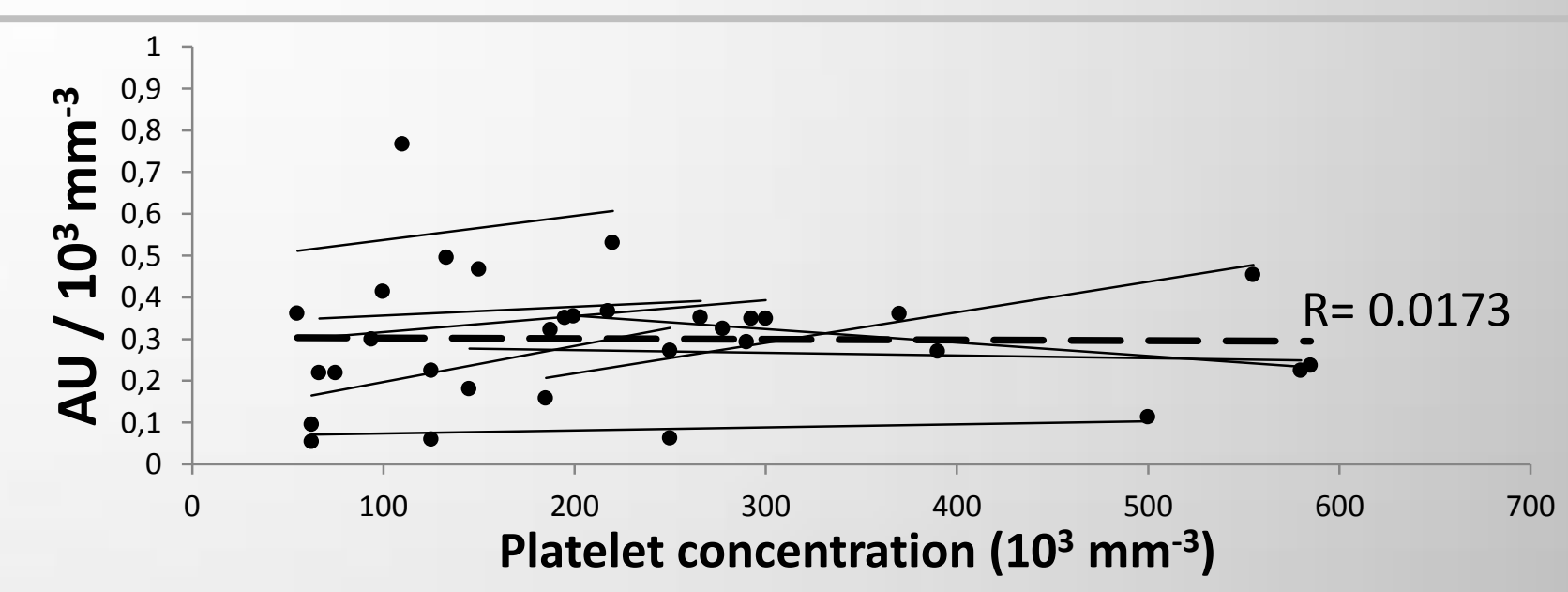

- Correlation between aggregation/platelet concentration ratio and platelet concentration with 32 data points from 8 individuals.

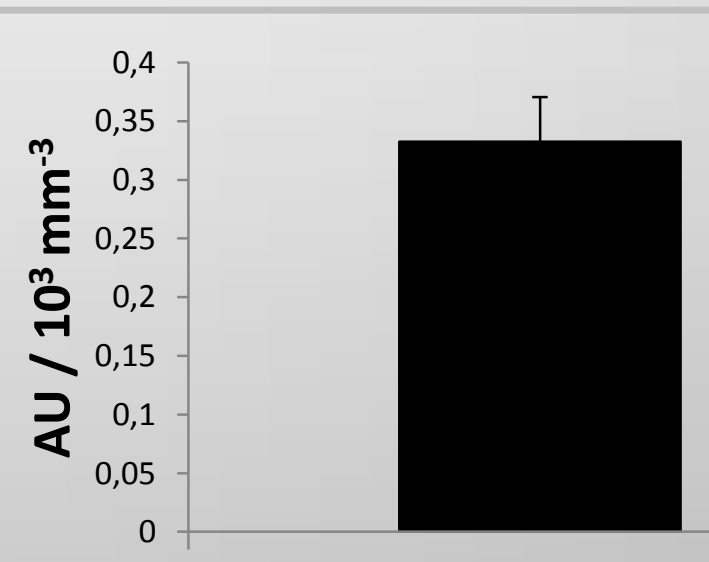

undiluted $p=0.22$

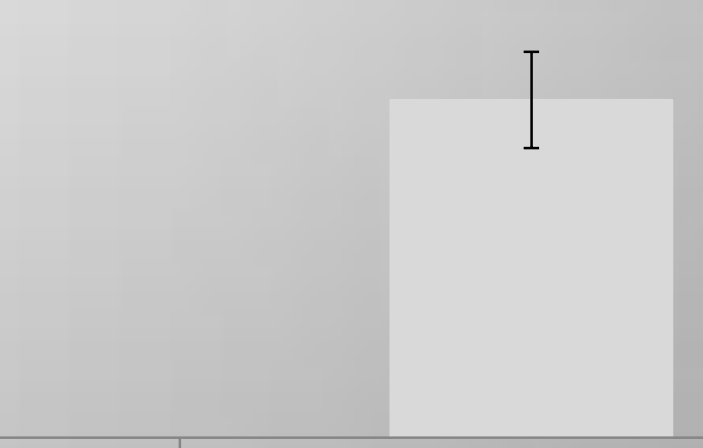

diluted 2 folds

- Comparison between aggregation/platelet concentration ratio in undiluted $n=19$ and twofold diluted platelet rich plasma $n=19$.

Conclusion: In individuals, there is a strict proportionality between platelet concentration and aggregation, and, therefore, this variable (aggregation/count ratio) might be indicative of the number of platelets aggregating when evoked by a particular activator. Conversely, normalization of impedance aggregometry amplitude can be achieved by simply dividing aggregation by platelet count.

References: 1. Siller-Matula, J Thromb Haemost. 2010 Feb;8(2): 351-9. 2. Boris T, Clinical Chemistry. 2007; 53:4, 614-619. 3. Müller-Schunk S. Am J Neuroradiol. 2008;16:786-791.

The work was supported by the the German academic exchange service (DAAD). 